\title{
Study on Shimmy Vibrations of Wheeled Casters
}

\author{
Kiyoshi Ioi, Yu Matsumoto, Seiki Nishikawa, Atsushi Suda, and Masaharu Tagami
}

\begin{abstract}
This paper focuses on the shimmy vibrations of wheeled casters. First, a new test caster is developed to freely change its mechanical properties. The accelerations of the caster are carefully measured by changing both the running velocity and the mechanical properties. Next, the running model of the caster is formulated on a horizontal plane, and the computer simulations are conducted based on the model. Then the acceleration data of the developed test caster are compared to those of the modeled caster in the computer, and it is confirmed that the simulation results have good agreements with the experimental results. Finally, some differences are discussed between the actual and computational data.
\end{abstract}

Index Terms-Wheeled caster, shimmy vibration, experiment, simulation.

\section{INTRODUCTION}

This paper reports the shimmy vibrations of wheeled casters. Wheeled casters have been used in the various movable products such as chairs, carts, and beds. However, it is well known that the heavy lateral vibrations of casters are often caused when there is some kind of relationship between the running velocity of the mover and the mechanical properties of the caster. The heavy lateral vibration is generally called shimmy. On the other hand, although these vibration problems have been studied around the world from long ago, the past obtained results are not yet sufficient for design engineering [1]-[7]. Moreover, most past results are obtained for not wheeled casters but wheeled tires with air. Hence, we report the recent research on the shimmy vibrations of the wheeled caster. First, an experimental test caster is newly designed to freely change the mechanical properties of the caster. The developed test caster is set up on an industrial running machine, and the accelerations of the caster are carefully measured by changing both the running machine's velocity and the caster's mechanical properties. Next, the simple running model of the caster is formulated on a horizontal plane, and many simulations are conducted by using the design parameters of the test caster. Then the acceleration data of the test caster are compared to those of the modeled caster in the simulation, and it is confirmed that the simulation results have good agreements with the experimental results in the view of the frequencies and amplitudes of

Manuscript received October 3, 2014; revised January 12, 2015.

Kiyoshi Ioi, Yu Matsumoto, and Seiki Nishikawa with Kinki University, Faculty of Science and Engineering, Osaka, Japan (e-mail: ioi@mech.kindai.ac.jp, 1333306068m@kindai.ac.jp, 1110350039r@kindai.ac.jp).

Atsushi Suda is with National Institute of Technology Maizuru College, Mechanical Engineering, Kyoto, Japan (e-mail: a.suda@maizuru-ct.ac.jp).

Masaharu Tagami is with Kinki University, Faculty of Engineering, Hiroshima, Japan (e-mail: tagami@ hiro.kindai.ac.jp). acceleration. Additionally, some differences are discussed between the actual and simulation data. Finally, we summarize the main results and propose the model improvements.

\section{BASIC DESIGN OF TEST CASTER}

\section{A. Variable Mechanism of Test Caster}

The test caster is designed to change the height, the eccentric length, and the mass of the fork as shown in Fig. 1. Most materials of the caster are steels, and the outside circle of the wheel is made from urethane gum. Here, what is called the fork is placed between the vertically rotational axis and the wheel axis, and is a strength component of the caster to support the cart load. The test caster has four holes to change the height from the rotational center to the wheel center. The eccentric length is also able to be changed by moving the part with the rotational axis along the long hole and then fastening on the fork. The horizontal seven holes shown in Fig. 1 are used to fasten a tuned weight, and the total mass of the caster is tuned by replacing the weight and positioning on the fork. Table I shows the specifications of the test caster.

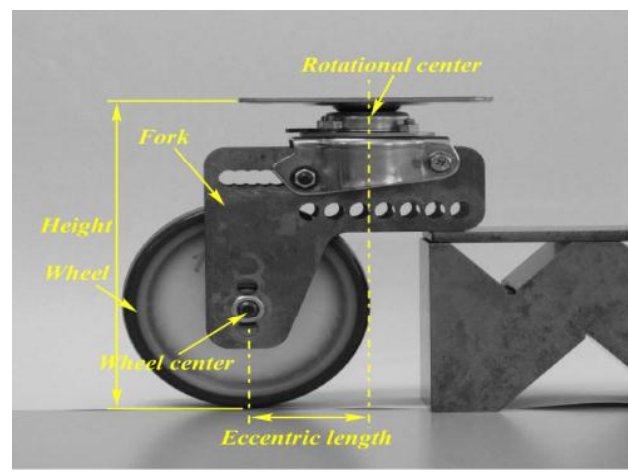

Fig. 1. Overview of test caster.

TABLE I: SPECIFICATIONS OF TEST CASTER

\begin{tabular}{|c|c|}
\hline Mass $[\mathrm{g}]$ & 709 \\
\hline Height $[\mathrm{mm}]$ & $130-160$ \\
& (Tuning in 10mm intervals) \\
\hline Eccentric length $[\mathrm{mm}]$ & $\begin{array}{c}20-50 \\
\text { (Tuning in 5mm intervals) }\end{array}$ \\
\hline Wheel diameter $[\mathrm{mm}]$ & 100 \\
\hline
\end{tabular}

\section{B. Setup of Disturbance Generator}

An industrial running machine is used to simulate the running state of the test caster on behalf of actual roads. The running machine is a roller typed one, and a pendulum is attached to the upper side of the machine in order to give the test caster a force disturbance from its outside. The force disturbance to the caster is nearly kept constant since the pendulum is always released from the same position. Fig. 2 shows the running machine with the pendulum. This 
machine is able to change the running speed and the strength of force disturbance. The test caster is attached to a cart board with an appropriate mass, and is set on the roller road. The cart board is also supported by vertical sliding bearings on both sides to simulate the vertical free motion of the cart board.

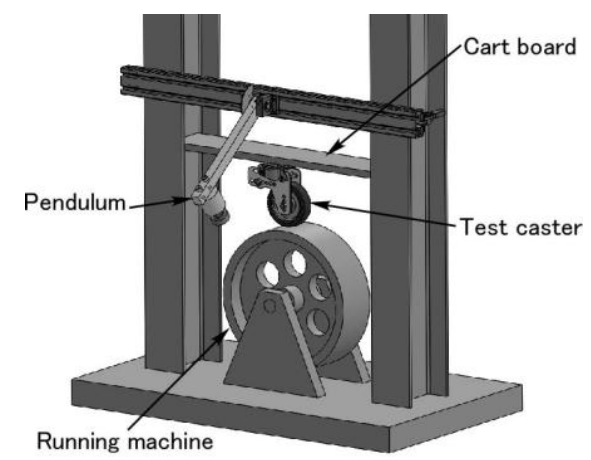

Fig. 2. Running machine with pendulum disturbance.

\section{Dynamical Modeling of TeSt CASTER}

\section{A. Modeling of Caster on Horizontal Plane}

The dynamical model of the caster is basically formulated on a horizontal plane in order to simulate the heavy lateral vibrations what is called shimmy. However, the derived model partially includes the bending and torsional effects of the fork material dependent on Young's modulus. Fig. 3 shows the caster model on a horizontal plane. Point A corresponds to the rotational center of the caster, and is assumed to be pulled by pushing a cart board along the ${ }^{0} x$ direction axis. Point $\mathrm{C}$ denotes the mass center of the caster including the wheel mass, and Point $\mathrm{P}$ is the contact point of the caster on the horizontal plane.

First, the basic 2-dimensional motion equation is as follows according to Newton-Euler's formulation:

$$
\begin{aligned}
m_{C} \ddot{x}_{C} & =F_{a x}+F_{C} \sin (\beta+\theta)-\operatorname{sgn}\left[\dot{x}_{C}\right] \mu_{R} W \cos \theta, \\
m_{C} \ddot{y}_{C} & =F_{a y}-F_{C} \cos (\beta+\theta)-\operatorname{sgn}\left[\dot{y}_{C}\right] \mu_{R} W \sin \theta, \\
I_{C} \ddot{\theta}= & r_{a}\left(F_{a y} \cos \theta-F_{a x} \sin \theta\right)+r_{p} F_{C} \cos \beta \\
& -c_{A} \dot{\theta}-\operatorname{sgn}[\dot{\theta}] \mu_{\theta} N_{W} .
\end{aligned}
$$

Here, Symbol $\operatorname{sgn}[*]$ denotes a sign function that has 1 or -1 corresponding to positive or negative, respectively. Other Symbols are defined as follows:

$\left(x_{C}, y_{C}\right)$ : Position of gravity center $\mathrm{C}$,

$\theta$ : Yawing angle of caster,

$\beta$ : Lateral sliding angle of wheel,

$r_{a}$ : Length between gravity center $\mathrm{C}$ and point $\mathrm{A}$,

$r_{p}$ : Length between gravity center $\mathrm{C}$ and point $\mathrm{P}$,

$m_{C}$ : Mass of caster with wheel,

$I_{C}$ : Moment of inertia about gravity center $\mathrm{C}$,

$\left(F_{a x}, F_{a y}\right):$ Constrained force at Point A,

$F_{C}$ : Cornering force dependent on angle $\beta$ at Point $\mathrm{P}$,

$W$ : Vertical weight against wheel at Point $\mathrm{P}$,

$\mu_{R}$ : Frictional coefficient of rolling motion between wheel and road at Point $\mathrm{P}$,

$c_{A}$ : Viscous coefficient about rotational axis at Point A,
$N_{W}$ : Frictional moment proportional to vertical weight, $\mu_{\theta}$ : Coulomb's frictional coefficient about rotational axis at Point A.

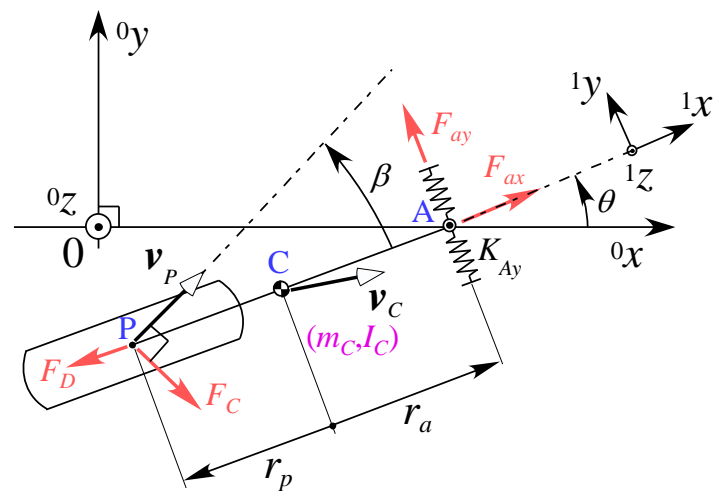

Fig. 3. Caster model on horizontal plane.

Moreover, the velocity $v_{P}=\left(\dot{x}_{P}, \dot{y}_{P}\right)$ at Point $\mathrm{P}$ has the following relationship as a nonholonomic constraint:

$$
\tan (\beta+\theta)=\frac{\dot{y}_{P}}{\dot{x}_{P}} .
$$

Here, $\dot{x}_{P}=\dot{x}_{C}+r_{p} \sin \theta \dot{\theta}, \quad \dot{y}_{P}=\dot{y}_{C}-r_{p} \cos \theta \dot{\theta}$.

The above cornering force $F_{C}$ in Equation (1) is approximated as follows considering the force saturation:

$$
F_{C} \cong K \beta, \quad F_{C \max }=\mu_{S} W .
$$

Here, symbols $\mu_{S}$ and $K$ denote the frictional coefficient and the cornering power, respectively, and they are usually determined by experiments [8], [9].

Although the constrained force $F_{a x}$ is exactly a ${ }^{1} x$ direction force at Point $\mathrm{A}$, the force is considered to be equivalent to the pulling force of the caster at Point $\mathrm{A}$ when the yawing angle $\theta$ is small enough. For this reason, the force $F_{a x}$ is used as the input force pulling Point $\mathrm{A}$ in the simulation based on Equation (1).

On the other hand, the constrained force $F_{a y}$ is not yet clarified. Hence, the inner force $F_{a y}$ is assumed to be caused by the bending and torsional elasticity of the fork dependent on both the height and eccentric length from the rotational axis to Point P. From the assumption, when the bending displacement $y_{A}$ at Point $\mathrm{P}$ is small enough about the fork, the inner force $F_{a y}$ is expressed as follows by using the one spring constant $K_{A y}$ equivalent to the combinational of bending and torsional stiffness:

$$
F_{a y}=-K_{A y} y_{A} \text {, where } y_{A}=y_{C}+r_{a} \sin \theta .
$$

Here, the value of $K_{A y}$ is estimated by using the torsional stiffness $K_{A, P 1}$ of horizontal part and the bending stiffness $K_{P 1, P 3}$ of vertical part about the whole caster. The formula of spring constant is well known as follows:

$$
\frac{1}{K_{A y}}=\frac{1}{K_{A, P_{1}}}+\frac{1}{K_{P_{1}, P_{3}}} .
$$

The values of $K_{A, P 1}$ and $K_{P 1, P 3}$ are also calculated by applying some basic formulas of material mechanics.

Fig. 4 shows the relationship between the stiffness and the displacement $y_{A}$. 


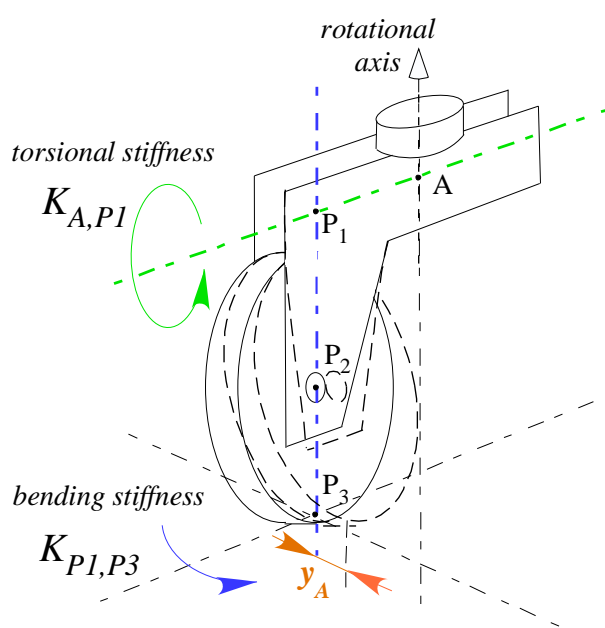

Fig. 4. Relationship between displacement and stiffness.
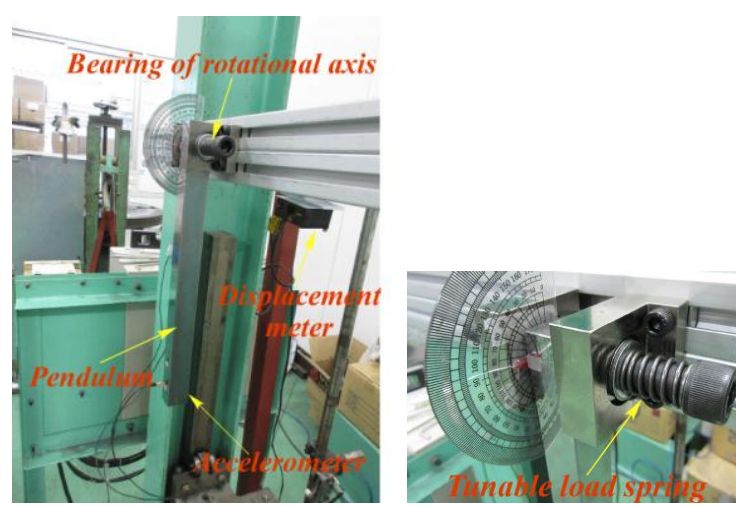

Fig. 5. Measuring system of parameters about rotational axis.

\section{EXPERIMENT AND SIMULATION}

\section{A. Measurement of Basic Parameters}

Some parameters for simulation are difficult to be determined by the mechanical design parameters. Their parameters are symbols $\mu_{R}, c_{A}, \mu_{\theta}, \mu_{S}$, and $K$. The frictional coefficient of rolling motion $\mu_{R}$ is expected to be negligibly small, compared to parameters $c_{A}$ and $\mu_{\theta}$. The important parameters $c_{A}$ and $\mu_{\theta}$ about the rotational axis are measured by using the pendulum motion in the gravity field. Fig. 5 shows the measuring system of the parameters. The vertical weight loading on the caster axis is physically simulated because the rotational axis of the pendulum is always pressed by the tunable load spring. The acceleration of the pendulum is measured by an accelerometer attached to the pendulum, and the parameters $c_{A}$ and $\mu_{\theta}$ are estimated by applying RMS method to the time sequential data of accelerations and velocities obtained by time integration of accelerations. The accelerations and velocities are applied to RMS in the neighborhood of the vertical position of the pendulum to avoid the effect of the gravitational moment. On the other hand, the parameters $\mu_{s}$ and $K$ between the caster wheel and the running road are measured by different methods, respectively. The frictional coefficient $\mu_{s}$ is estimated by a specific pulling device for measurement of static forces. The cornering power $K$ is estimated from the contact area between the wheel and a flat plate, and the value of $K$ is calculated by the basic shearing formula of material mechanics. Fig. 6 shows the contact areas between the wheel and the flat plate when the vertical weight changes against the caster wheel. Pink inks are painted on the wheel to stamp the contact area clearly on the plate covered with a white sheet. We can see that the contact area becomes a circular shape since the outside of the test wheel has a barrel shape.

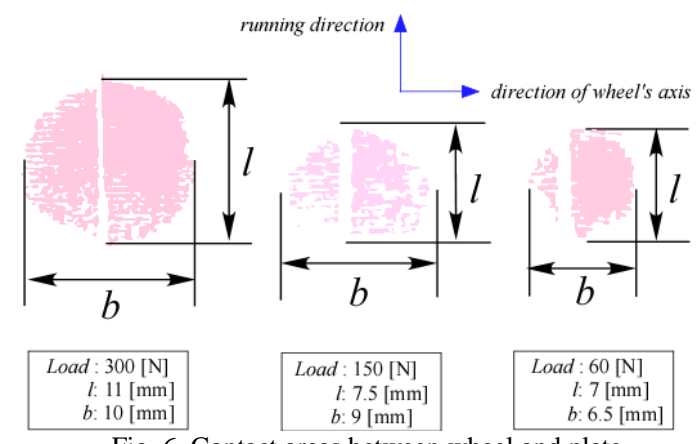

Fig. 6. Contact areas between wheel and plate.

\section{B. Comparison of Shimmy Vibrations}

The developed test caster mentioned in Section II changes both the mass center of the caster and the moment of inertia about its center according to the positioning of the vertically rotational center and the wheel center. At the start, the height $P_{1} P_{2}$ in Fig. 4 is fixed from the rotational center to the wheel center, and the only eccentric length $\left(r_{a}+r_{p}\right)$ in Fig. 2 is variable. Table II shows the length from the rotational center to the mass center and the moment of inertia about the mass center when the eccentric length changes under the fixed height of $150[\mathrm{~mm}]$. These values on Table II are estimated by using the automatic calculation tool of a commercial 3D-CAD 'Solid-Works ${ }^{\mathrm{TM}}$ '. To observe the shimmy vibrations of caster, an accelerometer is attached to the near side of the rotational center not to be affected by the bending and torsional elasticity of the fork.

TABLE II: RELATIONSHIP OF ECCENTRIC LENGTH AND MASS PROPERTIES

\begin{tabular}{|c|c|c|c|}
\hline case & $\begin{array}{c}\text { Eccentric length } \\
\left(r_{a}+r_{p}\right)[\mathrm{m}]\end{array}$ & $\begin{array}{c}\text { Length from the } \\
\text { rotational center } \\
\text { to the mass center } \\
r_{a}[\mathrm{~m}]\end{array}$ & $\begin{array}{c}\text { Moment of } \\
\text { inertia about } \\
\text { the mass center } \\
I_{C}\left[\mathrm{kgm}^{2}\right]\end{array}$ \\
\hline 1 & 0.020 & 0.00371 & 0.0004495 \\
\hline 2 & 0.025 & 0.00685 & 0.0004511 \\
\hline 3 & 0.030 & 0.00999 & 0.0004585 \\
\hline 4 & 0.035 & 0.01313 & 0.0004717 \\
\hline 5 & 0.040 & 0.01672 & 0.0004908 \\
\hline 6 & 0.045 & 0.01941 & 0.0005158 \\
\hline 7 & 0.050 & 0.02254 & 0.0005466 \\
\hline
\end{tabular}

Table III shows the simulation parameters estimated from the experimental measurements.

TABLE III: MEASURED PARAMETERS FOR SIMULATION

\begin{tabular}{|c|c|r|}
\hline & Symbol & \multicolumn{1}{|c|}{ Value } \\
\hline Vertical weight [N] & $W$ & 57.7 \\
\hline Equivalent spring constant [N/m] & $K a y$ & 32400 \\
\hline Viscous frictional coefficient[Nms] & $C_{A}$ & 0.0139 \\
\hline Rolling frictional coefficient & $\mu_{R}$ & 0.00784 \\
\hline Coulomb's frictional coefficient & $\mu_{\theta}$ & 0.00128 \\
\hline Cornaring power [N/rad] & $K$ & 34400 \\
\hline Frictional coefficient & $\mu_{S}$ & 0.34 \\
\hline
\end{tabular}

Fig. 7, Fig. 8, and Fig. 9 show the experimental results of 
the caster acceleration in the case- 1 , the case- 4 and case- 7 on Table II, respectively. It was confirmed that the shimmy vibration occurs when the speed of the running machine increases. Fig. 10, Fig. 11, and Fig. 12 show the simulation results of the caster acceleration using the design parameters corresponding to the experimental conditions. When the experimental results are compared to the simulation results, it is observed that the amplitude and vibration frequency of acceleration has a similar tendency with each other. In both the experiments and the simulations, the vibration amplitude and frequency increase when the eccentric length of the caster becomes longer according to seven cases shown on Table II. It is considered that the vibration frequency of the simulation is higher than that of the experiment because the estimated parameter $K_{A y}$ in Equation (5) is a little larger than the actual spring constant. However, the value of vibration frequency depends on not only the spring constant of the fork but also the running speed of the caster, because the natural frequency of the caster itself becomes lower when the eccentric length becomes longer. Accordingly, the vibration frequency must be carefully evaluated considering both the running speed and the fork stiffness. Furthermore, the amplitude of the shimmy acceleration also depends on the value of the force saturation $F_{\text {Cmax }}$ in Equation (3).

Here, the occurrence of shimmy vibration is newly defined as the case that the lateral vibrations of acceleration continue for more ten seconds after accepting the pendulum disturbance. The first and smallest running speed is named 'occurrence speed of shimmy', which causes the heavy lateral vibrations.

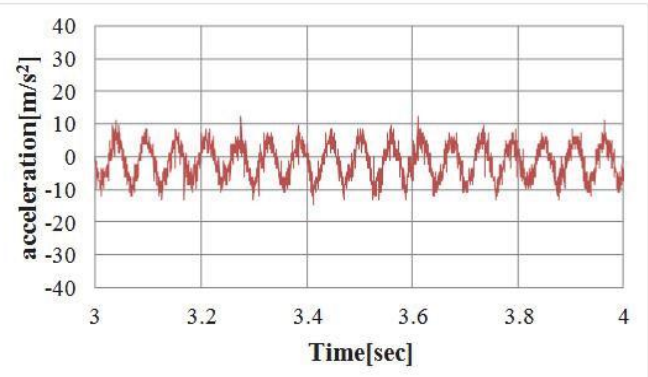

Fig. 7. Experimental result of shimmy vibration (Case-1).

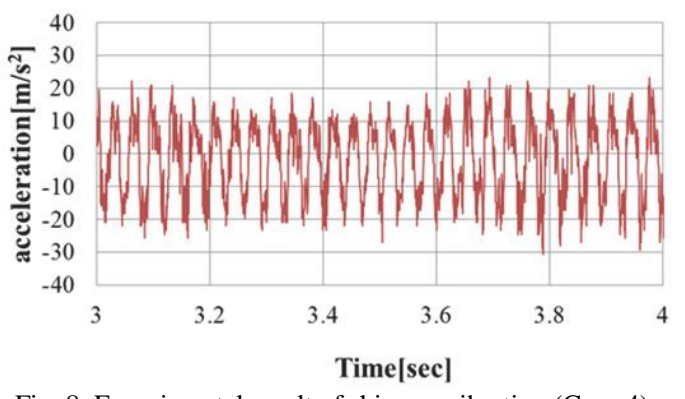

Fig. 8. Experimental result of shimmy vibration (Case-4).

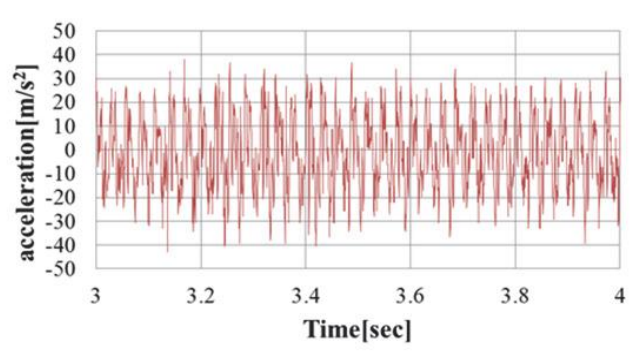

Fig. 9. Experimental result of shimmy vibration (Case-7).

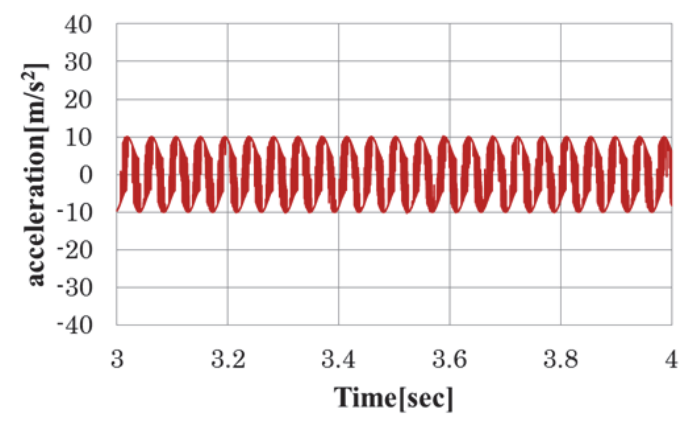

Fig. 10. Simulation result of shimmy vibration (Case-1).

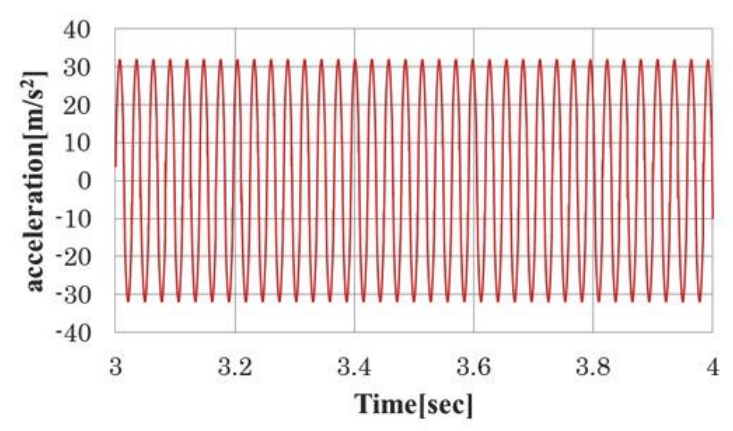

Fig. 11. Simulation result of shimmy vibration (Case-4).

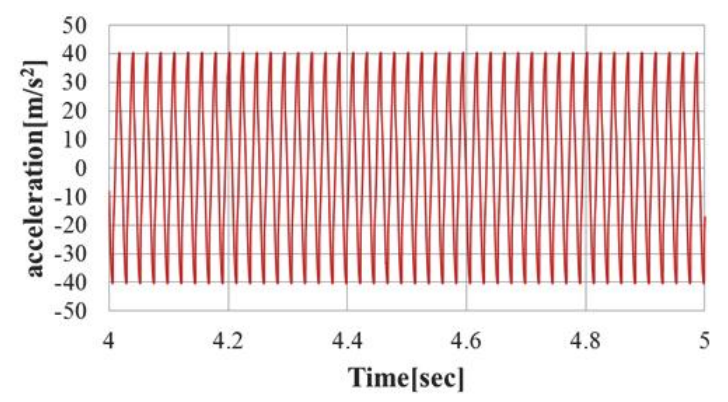

Fig. 12. Simulation result of shimmy vibration (Case-7).

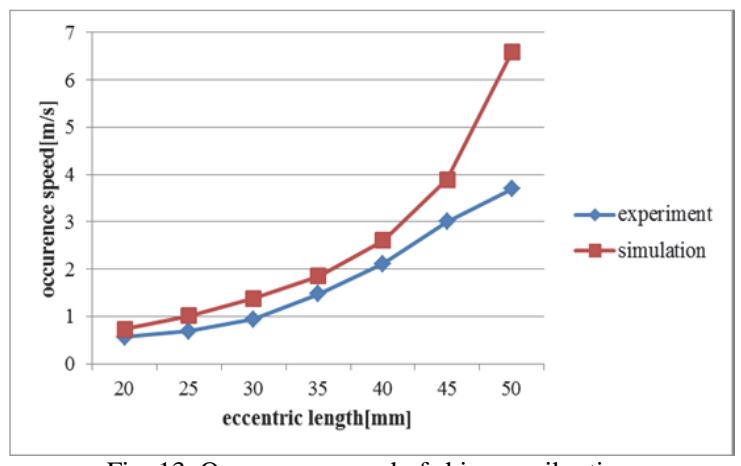

Fig. 13. Occurrence speed of shimmy vibration.

We investigated the relationship between the shimmy occurrence and the running speed when the eccentric length changes. Fig. 13 shows the relationship between the eccentric length and the occurrence speed of shimmy. Here, the blue plots and red plots denote the experimental and simulation results, respectively. The longer the eccentric length is, the larger the occurrence speed becomes nonlinearly. The simulation tendency of the occurrence speed is also similar to the experimental tendency of that except for the case 7. The error between the experiment and simulation increases when the eccentric length becomes long, and its disagreement is considered to be caused by the 
changed friction about the rotational axis. From these results, the proposed caster formulation is considered to become a suitable model for simulating the shimmy vibrations of actual cart casters.

\section{CONCLUSION}

This paper reported the shimmy vibrations of wheeled casters. A test caster was newly developed, and the experimental results were compared to the simulation results. The main results are as follows:

1) A test caster was designed to freely change its mechanical properties such as the eccentric length, the height, and the mass.

2) Many experimental results of the test caster were acquired by using an industrial running machine and a disturbance generator.

3) The running model of the caster was formulated on a horizontal plane, and many simulation results were obtained based on the model.

4) The experimental results had good agreements with the simulation results in the view of the frequency and amplitude of acceleration.

Although the simulation results had good agreements with the experimental results, it is a little difficult to determine the design parameters not to generate the shimmy vibrations, because some simulations have to be repeatedly conducted by human trials. Some kind of design chart is strongly required in order to develop the ideal and nonshimmy caster, which every engineer is able to simply deal with at the usual designing stage [10]. In near future, we would like to complete the design chart based on the comparisons of experiments and simulations. From many simulation results, it is confirmed that the shimmy vibrations largely change under frictional effects, and disappear or appear dependent on the frictional force about the rotational center and the cornering force at the contact point on roads. It is very important to precisely formulate the frictional model of wheeled caster. Moreover, the traditional wheeled caster may need some mechanical improvement in order to drastically restrict the shimmy vibrations. For this realization, the mechanical structure of caster must be newly designed from the view of dynamics and kinematics. Furthermore, a kind of actuator equipped with a simple controller is also considered to be placed at the rotational center of the caster to reduce the shimmy vibrations. Many future works are left unsolved to complete the ideal caster without lateral vibrations.

\section{REFERENCES}

[1] D. Takacs and Gabor Stepan, "Experimental study of the shimmy motion of wheels," in Proc. $22^{\text {nd }}$ DANUBIA-ADRIA Symposium on Experimental Methods in Solid Mechanics, 2005.

[2] D. Takacs, G. Stepan, and S. J. Hogan, "Bifurcations of a towed rigid wheel," in Proc. $6^{\text {th }}$ European Solid Mechanics Conference, 2006.

[3] D. Takacs and G. Stepan, "Experiments on quasiperiodic wheel shimmy," Journal of Computational and Nonlinear Dynamics, ASME Trans., vol. 4, 031007-1-7, 2009

[4] D. Takacs, G. Orosz, and G. Stepan, "Delay effects in shimmy dynamics of wheels with stretched string-like tyres," Europian Journal of Mechanics A/Solids, vol. 28, pp. 516-525, 2009.

[5] D. De Falco, G. Di Massa, and S. Pagano, "Experimental investigation on the shimmy phenomenon," in Proc. Bicycle and Motorcycle Dynamics, 2010.
[6] P. Thota, B. Krauskopf, and M. Lowenberg, "Shimmy a nonlinear model of an aircraft nose landing gear with non-zero rake angle," in Proc. $6^{\text {th }}$ EUROMECH Conference ENOC, 2008.

[7] C. Howcroft, B. Krauskopf, M. H. Lowenberg, and S. A. Neild, "Influence of variable side-stay geometry on the shimmy dynamics of an aircraft dual-wheel main landing gear," SIAM Journal of Applied Dynamical Systems, vol. 12, no. 3, pp. 1181-1209, 2013.

[8] M. Abe, Automotive Vehicle Dynamics Theory and Applications, Tokyo denki university press (in Japanese), 2012.

[9] S. Kobayashi: Vibration Engineering, Maruzen Publishing (in Japanese), 1994.

[10] Yuei Caster Co., Ltd, Yuei Caster Comprehensive Catalog, vol. 15, 2012.

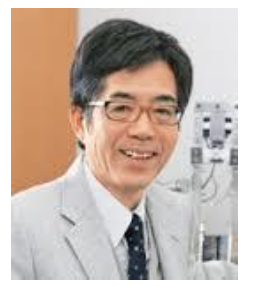

Kiyoshi Ioi received the B.S. degree and the M.S. degree in applied mathematics and physics engineering from the Kyoto University, Japan, in 1979 and 1981, respectively. He received the $\mathrm{Ph} . \mathrm{D}$. degree in applied system engineering from the Kyoto University, Japan, in 1991. He has been a professor at the Department of Mechanical Engineering, of the Faculty of Science and Engineering at Kinki University, Japan, since 2002. His research interests include broad aspects of mobile robotics, flying robotics, welfare mechatronics, vibration control, and human skill acquisition system.

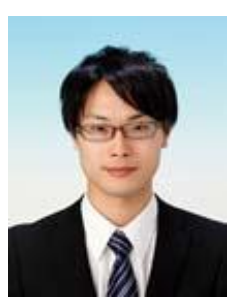

Yu Matsumoto received the B.S. degree in mechanical engineering from the Kinki University, Japan, in 2013. He is a student in M.S. degree's course at the graduate school of the Kinki University. He is interested in vibration problems of mobiles.

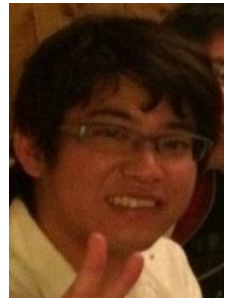

Seiki Nishikawa is a student in B.S. degree's course at mechanical engineering of the Kinki University, Japan, in 2014. He is interested in vibration problems of wheeled casters.

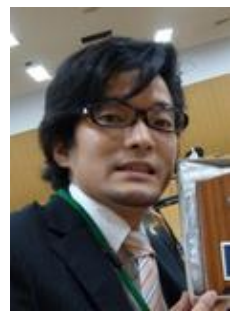

Atsushi Suda received the B.S. degree and the M.S. degree in mechanical engineering from the Kinki University, Japan, in 2005 and 2007, respectively. He received the Ph.D. degree in mechanical engineering from the Kinki University, Japan, in 2014. He has been a research associate at the Department of Mechanical Engineering, of National Institute of Technology Maizuru College, Japan, since 2014. His research interests include aspects of mechanical design, mobile vibrations, and mechanical control.

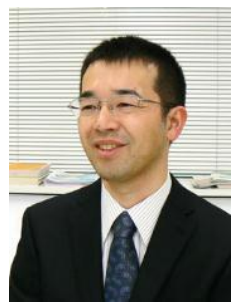

Masaharu Tagami received the B.S. degree and the M.S. degree in mechanical system engineering from the Kinki University, Japan, in 1999 and 2001, respectively. He received the $\mathrm{Ph} . \mathrm{D}$. degree in mechanical engineering from the Tokyo University of Agriculture and Technology, Japan, in 2008. He has been an assistant professor at the Department of Robotics, of the Faculty of Engineering at Kinki University, Japan, since 2014. His research interests include aspects in vibration control, robust control, and multi body mechanism. 